Virginia Commonwealth University

vCU Scholars Compass

2006

\title{
Orientational correlations in liquid acetone and dimethyl sulfoxide: A comparative study
}

Sylvia E. McLain

Rutherford Appleton Laboratories

Alan K. Soper

Rutherford Appleton Laboratory

Alenka Luzar

Virginia Commonwealth University

Follow this and additional works at: http://scholarscompass.vcu.edu/chem_pubs

Part of the Chemistry Commons

McLain, S. E., Soper, A. K., Luzar, A. Orientational correlations in liquid acetone and dimethyl sulfoxide: A comparative study. The Journal of Chemical Physics 124, 074502 (2006). Copyright (C) 2006 AIP Publishing LLC.

\section{Downloaded from}

http://scholarscompass.vcu.edu/chem_pubs/60

This Article is brought to you for free and open access by the Dept. of Chemistry at VCU Scholars Compass. It has been accepted for inclusion in Chemistry Publications by an authorized administrator of VCU Scholars Compass. For more information, please contact libcompass@vcu.edu. 


\title{
Orientational correlations in liquid acetone and dimethyl sulfoxide: A comparative study
}

\author{
Sylvia E. McLain ${ }^{\text {a) }}$ and Alan K. Soper \\ ISIS Facility, Rutherford Appleton Laboratories, Chilton, Didcot, Oxon OX11 0QX, United Kingdom
}

\author{
Alenka Luzar \\ Department of Chemistry, Virginia Commonwealth University, Richmond, Virginia 23284-2006
}

(Received 10 August 2005; accepted 4 January 2006; published online 16 February 2006)

\begin{abstract}
The structure of acetone and dimethyl sulfoxide in the liquid state is investigated using a combination of neutron diffraction measurements and empirical potential structure refinement (EPSR) modeling. By extracting the orientational correlations from the EPSR model, the alignment of dipoles in both fluids is identified. At short distances the dipoles or neighboring molecules are found to be in antiparallel configurations, but further out the molecules tend to be aligned predominately as head to tail in the manner of dipolar ordering. The distribution of these orientations in space around a central molecule is strongly influenced by the underlying symmetry of the central molecule. In both liquids there is evidence for weak methyl hydrogen to oxygen intermolecular contacts, though these probably do not constitute hydrogen bonds as such. (C) 2006 American Institute of Physics. [DOI: 10.1063/1.2170077]
\end{abstract}

\section{INTRODUCTION}

Understanding the relationships between the intermolecular interactions present in the liquid state is important in providing a complete picture of any solvent. Dimethyl sulfoxide (DMSO) and acetone are both common organic solvents with wide industrial applications. ${ }^{1}$ Their efficacy as solvents is predominately attributed to their polar aprotic nature where both liquids possess high dielectric constants (acetone $=20.7$ at $20{ }^{\circ} \mathrm{C}$; DMSO $=47.2$ at $20{ }^{\circ} \mathrm{C}$ ) and dipole moments $\left(\mu_{\mathrm{DMSO}}=3.96 \mathrm{D} ; \mu_{\text {acetone }}=2.88 \mathrm{D}\right.$ in the gas phase). ${ }^{2-4}$ Both molecules possess the same molecular groups differing only with respect to the central atom and as such the differences observed between acetone and DMSO, both with regard to the macroscopic properties as well as in the structure itself, are due to the electronic differences between sulfur and carbon.

In the gas phase DMSO and acetone adopt different geometries which are easily predicted by valence shell electron pair repulsion rules. ${ }^{5}$ Gaseous DMSO adopts a pyramidal $C_{s}$ geometry by virtue of a lone pair of electrons present on sulfur while acetone shows a planar $C_{2 \nu}$ geometry. Previous studies in the gas, liquid, and solid states show that this molecular geometry is conserved in all states for both molecules. ${ }^{6-15}$

The nature of the $\mathrm{S}-\mathrm{O}$ bond in DMSO has been the subject of several calculational investigations which show that a $\mathrm{S}^{+}-\mathrm{O}^{-}$formulation is the best representation, rather than a formal double $\mathrm{S}=\mathrm{O}$ bond. ${ }^{16-18}$ This formal charge separation will necessarily contribute strongly the dipole moment of the molecule. In contrast to DMSO, there is a formal double bond between $\mathrm{C}$ and $\mathrm{O}$ in acetone, with charge-

\footnotetext{
a) Author to whom correspondence should be addressed. Electronic mail: s.mclain@rl.ac.uk
}

separated resonance contributions playing only a small role in the ground-state description of the molecule. The difference between these two bonding regimes is supported by the $X-\mathrm{O}(X=\mathrm{S}, \mathrm{O})$ bond length, discussed below, as well as by the difference in the dielectric constant observed for these two molecules as higher charge separation in a bond generally gives rise to higher dielectric constants.

DMSO crystallizes in the monoclinic space group $P 2_{1} / C$ at $5 \mathrm{~K}$ and shows a S-O bond length of $1.531 \AA{ }^{19}$ which is shorter than the calculated single bond for DMSO but not sufficiently short to support a formal $\mathrm{S}=\mathrm{O}$ bond assignment. Acetone on the other hand crystallizes in the orthorhombic space group $\mathrm{Pbca}$ with a $\mathrm{C}=\mathrm{O}$ bond length of $1.21 \AA$ consistent with the $\mathrm{C}=\mathrm{O}$ double-bond formalism. ${ }^{9}$ In both crystalline DMSO and crystalline acetone, the molecules are layered along the $c$ axis aligned in a configuration that minimizes their dipole-dipole interactions. While DMSO molecules are aligned in an antiparallel fashion consistently throughout the crystal, the structure of acetone beyond the nearest-neighbor distance consists of perpendicular carbonyl interactions between each pair of antiparallel nearest neighbors. When lowering the temperature or applying pressure to acetone in the solid state, the antiparallel interactions are lost and only perpendicular carbonyl interactions are observed. This structural change which necessarily shortens the $\mathrm{C}=\mathrm{O} \cdots \mathrm{C}=\mathrm{O}$ and $\mathrm{C}-\mathrm{H} \cdots \mathrm{O}$ contacts between acetone molecules has been used to justify the broad heat-capacity transition (over $\sim 60 \mathrm{~K}$ ) observed in solid-state acetone. ${ }^{9,20}$

Several structural studies of both DMSO and acetone both as pure liquids and as components of mixtures have been performed by diffraction ${ }^{6-8,10,12,21-24}$ as well as simulation. ${ }^{10-12,25-45}$ DMSO has been the subject of the majority of these studies whereas the structure of pure acetone has not been as widely reported. DMSO is more widely 
studied because of its extensive application in many disciplines such as biochemistry, organic chemistry, and biophysics. $^{1,46,47}$

This study details the results of neutron diffraction measurements on acetone and DMSO in the liquid state. Additionally computer simulation by empirical potential structure refinement (EPSR) has been employed to model the diffraction data. ${ }^{48}$ Given the large number of interatomic distances in these molecules, it is difficult to build a complete threedimensional picture of either liquid through experiment alone. Using this combination of neutron diffraction measurements coupled with the EPSR method orientational correlation functions have been extracted from the resulting model which are consistent with the measured data for both liquids. $^{48}$

\section{NEUTRON DIFFRACTION MEASUREMENTS}

\section{A. Theory}

Neutron diffraction with isotopic substitution is the premier technique by which the structure of molecular liquids containing hydrogen has been determined. ${ }^{49-57}$ This is primarily due to the lack of correlation between the atomic number and the strength of the nuclear scattering interaction where light atoms, such as hydrogen, have scattering intensities on the same order of magnitude as heavier elements. ${ }^{58}$ The quantity measured in a neutron diffraction experiment is the differential scattering cross section, $d \sigma / d \Omega$,
TABLE I. Weighting factor for partial structure factors present in measured DMSO and acetone samples where $X=\mathrm{S}$ for DMSO and $X=\mathrm{C}_{c}$ for acetone.

\begin{tabular}{ccccc}
\hline \hline & $d_{6}$-DMSO & $d_{6}$-acetone & $h_{6}$-acetone & $0.5 h_{6}: 0.5 d_{6}$-acetone \\
\hline $\mathrm{H}-\mathrm{H}$ & 0.4170 & 0.3704 & 0.2202 & 0.0645 \\
$\mathrm{H}-\mathrm{C}$ & 0.2771 & 0.2460 & 0.2609 & 0.1961 \\
$\mathrm{H}-X$ & 0.0750 & 0.1230 & 0.1305 & 0.0981 \\
$\mathrm{H}-\mathrm{O}$ & 0.1211 & 0.1075 & 0.1138 & 0.0855 \\
$\mathrm{C}-\mathrm{C}$ & 0.0457 & 0.0409 & 0.0775 & 0.1484 \\
$\mathrm{C}-X$ & 0.0198 & 0.0409 & 0.0775 & 0.1484 \\
$\mathrm{C}-\mathrm{O}$ & 0.0401 & 0.0356 & 0.0674 & 0.1291 \\
$X-X$ & 0.0002 & 0.0102 & 0.0193 & 0.0369 \\
$X-\mathrm{O}$ & 0.0009 & 0.0178 & 0.0337 & 0.0646 \\
$\mathrm{O}-\mathrm{O}$ & 0.0009 & 0.0008 & 0.0149 & 0.0285 \\
\hline \hline
\end{tabular}

$$
\frac{d \sigma}{d \Omega}=\frac{d \sigma}{d \Omega_{\text {self }}}+\frac{d \sigma}{d \Omega_{\text {distinct }}}=\sum_{\alpha} c_{\alpha} b_{\alpha}^{2}(1+P(Q, \theta))+F(Q),
$$

where $P(Q, \theta)$ is the inelastic contribution and $F_{N}(Q)$ is the total scattering structure factor arising from the "distinct scattering" contribution, $c_{\alpha}$ is the atomic fraction, and $b_{\alpha}$ the scattering length of isotope $\alpha . F(Q)$ is the sum of all FaberZiman partial structure factors, $S_{\alpha \beta}(Q)$, present in the sample weighted by their composition and scattering intensity. $F(Q)$ and $S_{\alpha \beta}(Q)$ are related by the following equation:

$$
F(Q)=\sum_{\alpha \beta \geqslant \alpha}\left(2-\delta_{\alpha \beta}\right) c_{\alpha} c_{\beta} b_{\alpha} b_{\beta}\left(S_{\alpha \beta}(Q)-1\right),
$$

where $Q$ is the magnitude of the change in momentum vector by the scattered neutrons and $Q=4 \pi \sin \theta / \lambda$. For both acetone and DMSO, $F(Q)$ can be written as

$$
\begin{aligned}
F(Q)= & b_{\mathrm{H}}^{2} c_{\mathrm{H}}^{2}\left[S_{\mathrm{HH}}(Q)-1\right]+2 b_{\mathrm{H}} b_{\mathrm{O}} c_{\mathrm{H}} c_{\mathrm{O}}\left[S_{\mathrm{HO}}(Q)-1\right]+2 b_{\mathrm{H}} b_{X} c_{\mathrm{H}} c_{X}\left[S_{\mathrm{HX}}(Q)-1\right]+2 b_{\mathrm{H}} b_{\mathrm{C}} c_{\mathrm{H}} c_{\mathrm{C}}\left[S_{\mathrm{HC}}(Q)-1\right] \\
& +2 b_{\mathrm{O}} b_{X} c_{\mathrm{O}} c_{X}\left[S_{\mathrm{OX}}(Q)-1\right]+2 b_{\mathrm{C}} b_{X} c_{\mathrm{C}} c_{X}\left[S_{\mathrm{CX}}(Q)-1\right]+2 b_{\mathrm{O}} b_{\mathrm{C}} c_{\mathrm{O}} c_{\mathrm{C}}\left[S_{\mathrm{OC}}(Q)-1\right]+b_{\mathrm{O}}^{2} c_{\mathrm{O}}^{2}\left[S_{\mathrm{OO}}(Q)-1\right] \\
& +b_{\mathrm{C}}^{2} c_{\mathrm{C}}^{2}\left[S_{\mathrm{CC}}(Q)-1\right]+b_{X}^{2} c_{X}^{2}\left[S_{X X}(Q)-1\right],
\end{aligned}
$$

where $X=\mathrm{S}$ for DMSO and $X=\mathrm{C}_{\mathrm{C}}$, signifying the carbonyl carbon, for acetone. The neutron weights outside each partial structure factor for each sample are shown in Table I. In order to show the relative intensity of scattering from each partial structure factor with respect to one another the weighting factors shown in the table have been normalized by dividing each by the sum of the total scattering in the sample, $\Sigma_{\alpha, \beta} c_{\alpha} c_{\beta} b_{\alpha} b_{\beta}$.

The Fourier transform of any structure factor yields the associated radial distribution function, $G(r)$, which is the sum of the respective atom-atom radial distribution functions (RDF's), $g_{\alpha \beta}(r)$ 's, each weighted by concentration and scattering length of atomic species, $\alpha$ and $\beta$, present in the sample.

$S_{\alpha \beta}(Q)$ is related to the radial distribution function $g_{\alpha \beta}(r)$ via

$$
S_{\alpha \beta}(Q)=1+\frac{4 \pi \rho}{Q} \int r\left[g_{\alpha \beta}(r)-1\right] \sin (Q r) d r,
$$

where $\rho$ is the number density of the sample, 0.0819 and 0.0844 atoms $/ \AA^{-3}$ for acetone and DMSO, respectively. Fourier transformation of the measured total structure factor yields the total radial distribution function whereas the Fourier transformation of any partial structure factor yields the corresponding site specific RDF.

\section{B. Experiment}

In order to extract site-specific information in acetone and DMSO, neutron diffraction experiments were performed on isotopomers of the two pure fluids at $298 \pm 3 \mathrm{~K}$. All liquids $-d_{6}$-acetone, $h_{6}$-acetone, and $d_{6}$-DMSO-were pur- 
TABLE II. Isotopomers of acetone and DMSO measured on a SANDALS.

\begin{tabular}{ccc}
\hline \hline Sample & Acetone & Sample size $(\mathrm{mm})$ \\
\hline$d_{6}$-acetone & $\left(\mathrm{CD}_{3}\right)_{2} \mathrm{CO}$ & 2 \\
$h_{6}$-acetone & $\left(\mathrm{CH}_{3}\right)_{2} \mathrm{CO}$ & 1 \\
$0.5 h_{6}: 0.5 d_{6^{-}}$ & {$\left[\left(\mathrm{CH}_{3}\right)_{2} \mathrm{CO}\right]_{0.5}\left[\left(\mathrm{CD}_{3}\right)_{2} \mathrm{CO}\right]_{0.5}$} & 1 \\
acetone & $\mathrm{DMSO}$ & \\
\hline & $\left(\mathrm{CD}_{3}\right)_{2} \mathrm{SO}$ & 1 \\
\hline$d_{6}$-DMSO &
\end{tabular}

chased from Sigma/Aldrich chemical company and were used without further purification. The diffraction data were obtained using the Small-Angle Neutron Diffractometer for Amorphous and Liquid Samples (SANDALS) located at the ISIS pulsed neutron facility at Rutherford Appleton Laboratory in the UK. SANDALS is an instrument well suited for structural measurements of liquids containing hydrogen with detectors that range from $3.9^{\circ}$ to $39^{\circ}$, giving a $Q$ range from 0.15 to $50 \AA^{-1}$. In addition to the detectors, SANDALS is equipped with a transmission monitor which measures the total cross section of the sample being measured, $\sigma_{\text {trans }}$, relative to the incident beam. Each of the samples measured was contained in sample cells constructed from a Ti/Zr null alloy metal where each cell has a $1 \mathrm{~mm}$ wall thickness. The use of this alloy allows for minimal coherent scattering from the sample cell leading to a more tractable data analysis for the samples themselves. The samples measured are listed in Table II along with the size of the sample measured by neutron diffraction.

The diffraction data collected for $d_{6}$-DMSO have been previously reported along with two different isotopomers of DMSO, namely, $h_{6}$-DMSO and a $0.67 h_{6}: 0.34 d_{6}$-DMSO mixture in a combined neutron diffraction and moleculardynamics study. ${ }^{10}$ Although the $d_{6}$-DMSO data reported here have been previously collected they have been reanalyzed in the present work from the raw data to differential cross section. The $h_{6}$-DMSO and $0.67 h_{6}: 0.34 d_{6}$-DMSO data were also analyzed; however, it was clear in a subsequent analysis that both of the data sets collected from these two samples were probably contaminated with water impurities, and so have not been used in the present analysis. The water was detected from the presence of a small negative peak at $r=\sim 0.98 \AA$ in the Fourier transform of the data (the corresponding $\mathrm{C}-\mathrm{H}$ peak is at $\sim 1.08 \AA$ ) arising from the $\mathrm{O}-\mathrm{H}$ bond in water. A similar peak was not present in the $d_{6}$-DMSO sample. This feature of the older data was detected as a result of the present method of data interpretation using EPSR, and was not noticed with the earlier methods as in the previous study the intramolecular contribution to the diffraction pattern was subtracted prior to the analysis of the composite intermolecular radial distribution functions. ${ }^{10}$

For each measurement the raw data for each sample as well as for each sample container have been converted to differential scattering cross section, after correcting for absorption, multiple scattering, and inelasticity effects, by using a program, GUDRUN, which is a new version of the previous ATLAS suite of programs available at ISIS. ${ }^{59}$

The measurement of $\sigma_{\text {trans }}$ allows for the composition as well as the density of each sample to be confirmed. In each case the level of $d \sigma / d \Omega$ was checked after the application of corrections using GUDRUN by comparison with theoretical values based on the known density and composition. ${ }^{58}$ In each sample measured the level of scatter measured by the transmission monitor was approximately $\sim 10 \%$ below the expected level, likely due to machining uncertainties on the interior of the sample containers. For this reason in each diffraction pattern the effective thickness of each sample was adjusted until the scattering level was within $1 \%$ of the expected value.

\section{EPSR AND ORIENTATIONAL CORRELATION FUNCTION ANALYSES}

\section{A. Methods \\ 1. EPSR}

EPSR was used to model the diffraction data collected from isotopomeric samples of both liquids. EPSR is a computational method created for modeling disordered materials such as liquids and glasses, ${ }^{48,50}$ which allows the reconstruction of orientational correlation functions from a set of onedimensional structure factor measurements in a manner which is consistent with the measured data.

EPSR begins with a standard Monte Carlo simulation using an initial reference potential where the potential consists of an intramolecular harmonic potential to define the geometry of the molecules being modeled, and an intermolecular potential, which, in the present case, consisted of Lennard-Jones 12-6 potentials for the site-site interactions on different molecules as well as Coloumbic interactions for some sites. This reference potential is used to generate a starting configuration of molecules. EPSR then iteratively adjusts a perturbation to this reference potential to obtain the best possible agreement between the computed $F(Q)$ and the experimental diffraction data. ${ }^{48,50}$

While EPSR provides a molecular ensemble which is consistent with the diffraction data measured, it does not necessarily provide a definitive model for the structure of the liquid in question. There may be several distinct structures which give an equally reasonable agreement between the data and simulation. This is especially true in the present case where there are many more partial structure factors than available diffraction contrasts. Therefore it is imperative that the simulation box be constrained from the outset with as much prior information regarding the properties of the liquid in question as is possible. For example, in the present study the large dipole moments and dielectric constants observed in liquid acetone and DMSO are important factors to consider when defining the reference potential.

The purpose of EPSR analysis in the present instance is not only to extract three-dimensional information from a model at the correct atomic number density which is consistent with the diffraction data but also to explore the validity of some potential models against a set of diffraction data. EPSR generates an effective site-site interaction potential which reproduces the measured diffraction data as close as possible. It has, however, so far proved impossible in EPSR to constrain both the energy and pressure in any reliable way 
as well as fit the diffraction data, so the simulation cannot be relied upon to reproduce the correct thermodynamics as well. Obtaining a fit to the measured data does not ensure the potential model is correct, but it is a necessary condition for any chosen potential model of the liquid. This direct comparison with the diffraction data in $Q$ space is rarely done with conventional molecular dynamics and Monte Carlo simulations of molecular liquids. Ideally a wide range of initial reference potentials should be explored, for example, those which include three-body or many-body forces such as polarizability. Unfortunately such a task is still beyond most computing strategies, and as such the most likely potentials must be selected from the literature for each individual case and tested against the experimental data.

Having found, through EPSR, a model liquid structure consistent with the diffraction data, it is useful to extract structural information from the simulation box concerning the intermolecular distributions, such as the individual sitesite RDF's, vide infra, as this information is not directly available from the experimental data. Because the site-site RDF's only give a one-dimensional representation of the fluid, it is difficult to use these distances to visualize the local spatial and orientational orders in three dimensions. For this reason, spatial density functions (SDF's) ${ }^{60,61}$ which allow a three-dimensional representation of the liquid structure to be constructed, were used to help determine the most probable nearest-neighbor positions for both fluids in the present study. Although the SDF's show the most probable location of nearest-neighbor molecules they do not necessarily give direct orientational information about the surrounding molecules. In light of this some aspects of the orientational paircorrelation function (OCF) are also shown. ${ }^{59}$ These tasks are achieved via spherical harmonic expansion of the full orientational pair-correlation function, ${ }^{62,63}$ using the simulation box to derive the positional and orientational coordinates of the molecules, and are described in more detail in the following section.

\section{Spatial density and orientational pair-correlation functions}

The details of the spherical harmonic expansion as well as the orientational correlation function calculation using a spherical harmonic expansion are given in detail elsewhere. ${ }^{62,63}$ Here a summary of these techniques which follow the notation used by Gray and Gubbins explicitly is presented. $^{63}$

A set of Euler angles within the laboratory reference frame $\omega_{M} \equiv\left(\varphi_{M} \theta_{M} \chi_{M}\right)$ for each molecule $M$ is calculated using a predefined set of molecular coordinate axes. The corresponding set of generalized spherical harmonic functions, $D_{m n}^{l}\left(\omega_{M}\right)$, are calculated for each molecule and for a range of $(l, m, n)$ values (up to $l=4$ in the present instance). The set of such functions is then correlated taking into account the relative position $\mathbf{r} \equiv\left(r, \omega_{L}\right) \equiv\left(r, \theta_{L} \varphi_{L}\right)$ of the second molecule with respect to the first, yielding a set of orientational correlation function expansion coefficients, $g\left(l_{1} l_{2} l ; n_{1} n_{2} ; r\right){ }^{63}$ From these coefficients the full orientational pair-correlation function is obtained as an expansion of the form

$$
\begin{aligned}
g\left(\mathbf{r}, \omega_{1}, \omega_{2}\right)= & \sum_{l_{1} l_{2} l} \sum_{m_{1} m_{2} m} \sum_{n_{1} n_{2}} g\left(l_{1} l_{2} l ; n_{1} n_{2} ; r\right) \\
& \times C\left(l_{1} l_{2} ; m_{1} m_{2} m\right) \\
& \times D_{m_{1} n_{1}}^{l_{1}}\left(\omega_{1}\right)^{*} D_{m_{2} n_{2}}^{l_{2}}\left(\omega_{2}\right)^{*} D_{m 0}^{l}\left(\omega_{L}\right),
\end{aligned}
$$

where $C\left(l_{1} l_{2} l ; m_{1} m_{2} m\right)$ are the Clebsch-Gordan coefficients, $\omega_{1}$ represents the Euler angles of molecule 1, $\omega_{2}$ represents the Euler angles of molecule 2 , and $\mathbf{r}=\left(r, \omega_{L}\right)$ represents the position of molecule 2 relative to molecule 1 in the laboratory coordinate frame.

In order to reconstruct the orientational correlation function it is convenient to set molecule 1 at the origin and orient it so that $\omega_{1}=0$. This serves to define the coordinate system about which the spatial density and orientation of second (neighboring) molecules will be plotted. It also leads to an immediate simplification of Eq. (5) in that $D_{m n}^{l}(000)$ $=\delta(m n)$, so that combining this with the requirement from the Clebsch-Gordan coefficients that $m=m_{1}+m_{2}$, the orientational pair-correlation function relative to a central molecule at the origin is given by

$$
\begin{aligned}
g\left(r, \omega, \omega_{M}\right)= & \sum_{l_{1} l_{2} l} \sum_{m} \sum_{n_{1} n_{2}} g\left(l_{1} l_{2} l ; n_{1} n_{2} ; r\right) \\
& \times C\left(l_{1} l_{2} l ; n_{1} m_{2} m\right) D_{m_{2} n_{2}}^{l_{2}}\left(\omega_{M}\right)^{*} D_{m 0}^{l}\left(\omega_{L}\right),
\end{aligned}
$$

where $m_{2}=m-n_{1}$. The spatial density function is generated by averaging the full orientational pair-correlation function over the orientations of the second molecule, $\omega_{M}$ $\equiv\left(\varphi_{M} \theta_{M} \chi_{M}\right)$, which immediately eliminates any terms in the summation shown in Eq. (6) for which $l_{2}, m_{2}, n_{2} \neq 0$. Hence the spatial density function is expressed as

$$
g(r, \omega)=\sum_{l_{1}} \sum_{n_{1}} g\left(l_{1} 0 l_{1} ; n_{1} 0 ; r\right) C\left(l_{1} 0 l_{1} ; n_{1} 0 n_{1}\right) D_{n_{1} 0}^{l_{1}}\left(\omega_{L}\right),
$$

from the closure relations for the Clebsch-Gordan coefficients $\left(l_{1}+l_{2} \geqslant l \geqslant\left|l_{1}-l_{2}\right|\right)$.

In general the full orientational pair-correlation function [Eq. (6)] is difficult to visualize because it is a function of six coordinates. To assist in this visualization, the spatial density function can be plotted to gauge the most likely places of finding neighboring molecules, then the orientational correlation function can be plotted for a specified $\omega_{L}$, after averaging over one of the remaining angular coordinates, e.g., $\chi_{M}$, as is done in the present work. This eliminates from Eq. (6) all terms for which $n_{2} \neq 0$, leaving an average orientational pair-correlation function which is a function of three variables, $r, \varphi_{M}$, and $\theta_{M}$, for a specified direction $\omega_{L} \equiv\left(\theta_{L} \varphi_{L}\right)$ away from the central molecule. Other averages of the orientational pair-correlation function over angular coordinates can be obtained by eliminating other terms in the full expression [Eq. (6)].

\section{B. Simulation}

EPSR simulations were performed for both acetone and DMSO by constructing a box containing 500 molecules at the appropriate density for each liquid. Table III shows the 
TABLE III. Intramolecular geometries used to construct the EPSR simulation boxes for acetone and DMSO.

\begin{tabular}{|c|c|c|c|c|}
\hline Acetone & & Bond ( & & Angle $\left(^{\circ}\right)$ \\
\hline & $\mathrm{H}-\mathrm{C}_{\mathrm{m}}$ & 1.10 & $\mathrm{H}-\mathrm{C}_{\mathrm{m}}-\mathrm{H}$ & 110.0 \\
\hline & $\mathrm{C}_{\mathrm{m}}-\mathrm{C}_{\mathrm{C}}$ & 1.50 & $\mathrm{C}_{\mathrm{m}}-\mathrm{C}_{\mathrm{C}}-\mathrm{C}_{\mathrm{m}}$ & 118.0 \\
\hline & $\mathrm{C}_{\mathrm{C}}-\mathrm{O}$ & 1.21 & $\mathrm{C}_{\mathrm{C}}-\mathrm{C}_{\mathrm{C}}-\mathrm{H}$ & 113.0 \\
\hline & & & $\mathrm{O}-\mathrm{C}_{\mathrm{C}}-\mathrm{C}_{\mathrm{m}}$ & 121.0 \\
\hline \multirow[t]{5}{*}{ DMSO } & & Bond $(\AA)$ & & Angle $\left(^{\circ}\right)$ \\
\hline & $\mathrm{H}-\mathrm{C}$ & 1.10 & $\mathrm{H}-\mathrm{C}-\mathrm{H}$ & 110.0 \\
\hline & $\mathrm{C}-\mathrm{S}$ & 1.74 & $\mathrm{C}-\mathrm{S}-\mathrm{C}$ & 99.0 \\
\hline & $\mathrm{S}-\mathrm{O}$ & 1.53 & $\mathrm{~S}-\mathrm{C}-\mathrm{H}$ & 111.0 \\
\hline & & & & 114.0 \\
\hline
\end{tabular}

intramolecular angles and distances used for the input molecules for each set of EPSR simulations. In each simulation, the methyl hydrogens were allowed to freely rotate around the $\mathrm{C}_{\mathrm{C}}-\mathrm{C}_{\mathrm{m}}$ axis in acetone and the $\mathrm{S}-\mathrm{C}$ axis in DMSO.

For acetone, the three measured structure factors were simultaneously fit using the acetone potential developed by Ferrario et $a l .{ }^{27}$ as the reference potentials, hereafter termed FHMK, as well as a potential developed by Wheeler and Rowley, ${ }^{40}$ hereafter termed WR. Both of these potentials are listed in Table IV where $\mathrm{C}_{\mathrm{m}}$ refers to the methyl carbons and $\mathrm{C}_{\mathrm{C}}$ refers to the central carbonyl carbon in acetone. Table IV also lists two separate potentials for DMSO, P1 and P2, developed by Luzar et al. ${ }^{10,11}$ which were used as reference potentials for EPSR fits to the DMSO diffraction data.

\section{RESULTS}

The total structure factor data for the three acetone measurements are shown in Fig. 1 along with the two EPSR fits to the data using the FHMK and WR reference potentials.

TABLE IV. Reference potentials used for initial input into EPSR fits to diffraction data for acetone and DMSO (Refs. 10, 11, 27, and 40).

\begin{tabular}{|c|c|c|c|c|}
\hline \multicolumn{5}{|c|}{ Acetone/FHMK (Ref. 27) } \\
\hline & $\varepsilon / \mathrm{kJ} \mathrm{mol}^{-1}$ & $\sigma(\AA)$ & $q_{e}$ & \\
\hline $\mathrm{H}$ & 0.0 & 0.0 & 0.0 & \\
\hline $\mathrm{C}_{\mathrm{m}}$ & 0.7605 & 3.88 & -0.032 & \\
\hline $\mathrm{C}_{\mathrm{C}}$ & 0.439 & 3.75 & 0.566 & \\
\hline $\mathrm{O}$ & 0.878 & 2.96 & -0.502 & \\
\hline \multicolumn{5}{|c|}{ Acetone/WR (Ref. 40) } \\
\hline & $\varepsilon / \mathrm{kJ} \mathrm{mol}^{-1}$ & $\sigma(\AA)$ & $q_{e}$ & \\
\hline $\mathrm{H}$ & 0.0 & 0.0 & 0.0 & \\
\hline $\mathrm{C}_{\mathrm{m}}$ & 0.68156 & 3.88 & 0.03 & \\
\hline $\mathrm{C}_{\mathrm{C}}$ & 0.41559 & 3.78 & 0.48 & \\
\hline \multirow[t]{3}{*}{$\mathrm{O}$} & 0.7065 & 3.01 & -0.54 & \\
\hline & \multicolumn{2}{|l|}{ DMSO (Ref. 11) } & $\mathrm{P} 1$ & $\mathrm{P} 2$ \\
\hline & $\varepsilon / \mathrm{kJ} \mathrm{mol}^{-1}$ & $\sigma(\AA)$ & $q_{e}$ & $q_{e}$ \\
\hline $\mathrm{H}$ & 0.0 & 0.0 & 0.0 & 0.0 \\
\hline $\mathrm{C}$ & 1.23 & 3.20 & 0.0 & 0.160 \\
\hline $\mathrm{S}$ & 0.99741 & 3.40 & 0.54 & 0.129 \\
\hline $\mathrm{O}$ & 0.29922 & 2.80 & -0.54 & -0.459 \\
\hline
\end{tabular}
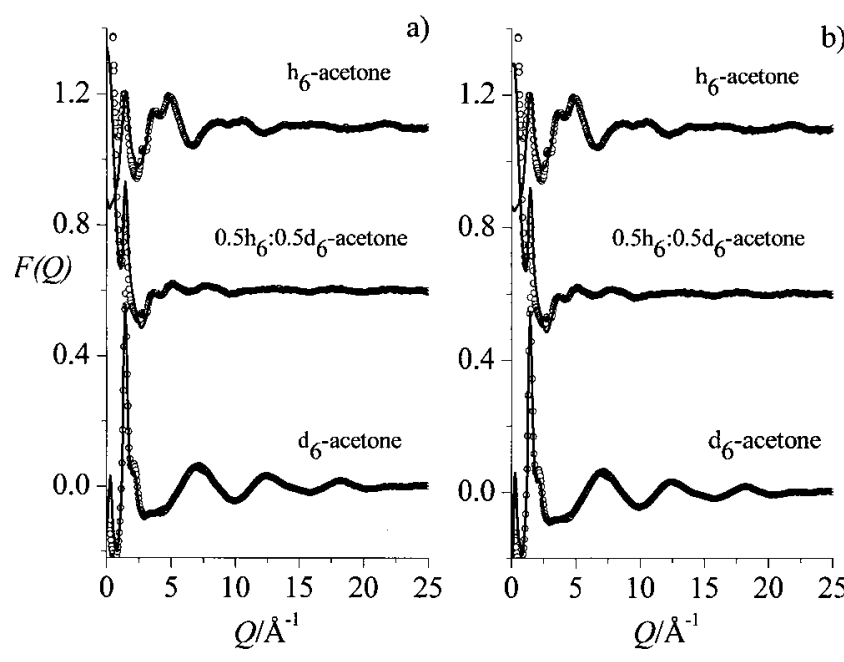

FIG. 1. $F(Q)$ data (circles) for $d_{6}$-acetone, $0.5 h_{6}: 0.5 d_{6}$-acetone, and $h_{6}$-acetone at $T=298 \mathrm{~K}$ and subsequent EPSR fits (solid lines) to the data using the (a) FHMK reference potential (Ref. 27) and (b) the WR reference potential (Ref. 40). The $0.5 h_{6}: 0.5 d_{6}$-acetone data have been shifted by 0.6 and the $h_{6}$-acetone data have been shifted by 1.1 in each case for clarity.

Clearly, both the WR and FHMK reference potentials provide good starting points for the EPSR fits to the three data sets measured. The only exception is at low values of $Q(Q$ $<3 \AA^{-1}$ ) where the background and inelasticity corrections to the data are most difficult to remove when the isotopomers contain light hydrogen, as is the case with the $h_{6}$-acetone and the 50:50 mixture. Although the starting potentials are different, the fits to the data show no obvious differences, indicating that the EPSR procedure is able to counteract differences between the two reference potentials with regard to reproducing the structure. Figure 2 shows the site-site RDF's obtained from both of these models and as expected from the fits in Fig. 1, there are negligible differences in these functions between the two fits. Additionally, Table V shows the coordination numbers for each RDF shown in Fig. 2.

The total structure factor measurement for $d_{6}$-DMSO

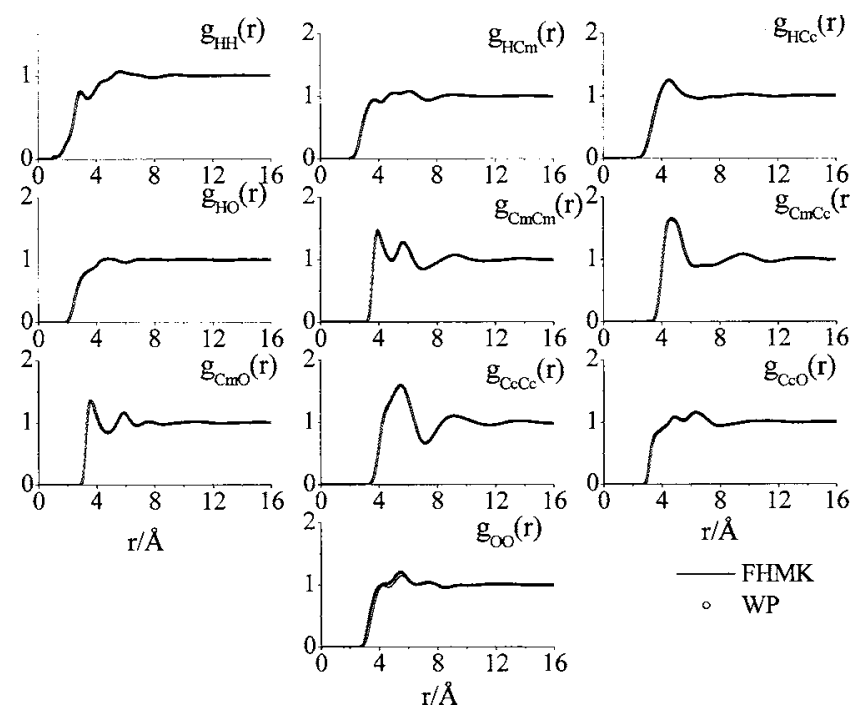

FIG. 2. Site-site intermolecular pair-correlation functions for acetone generated by EPSR fits to the data using the FHMK and WR reference potentials (Refs. 27 and 40). 
TABLE V. Coordination numbers for site-site radial distribution functions from EPSR fits to acetone using the FHMK reference potential (Ref. 27).

\begin{tabular}{ccr}
\hline \hline $\mathrm{RDF}$ & $r(\AA)$ & $\mathrm{CN}$ \\
\hline $\mathrm{C}_{\mathrm{C}}-\mathrm{C}_{\mathrm{C}}$ & 7.14 & 12.1 \\
$\mathrm{O}-\mathrm{C}_{\mathrm{C}}$ & 5.54 & 4.7 \\
& 7.74 & 15.4 \\
$\mathrm{C}_{\mathrm{m}}-\mathrm{C}_{\mathrm{C}}$ & 6.46 & 8.8 \\
$\mathrm{H}-\mathrm{C}_{\mathrm{C}}$ & 6.04 & 6.9 \\
$\mathrm{H}-\mathrm{H}$ & 7.72 & 90.0 \\
$\mathrm{H}-\mathrm{C}_{\mathrm{m}}$ & 4.16 & 3.0 \\
& 5.54 & 10.0 \\
& 7.38 & 26.2 \\
$\mathrm{H}-\mathrm{O}$ & 5.77 & 5.7 \\
$\mathrm{C}_{\mathrm{m}}-\mathrm{O}$ & 4.75 & 2.7 \\
& 6.76 & 9.8 \\
$\mathrm{O}-\mathrm{O}$ & 6.40 & 8.1 \\
& 8.53 & 20.6 \\
$\mathrm{C}_{\mathrm{m}}-\mathrm{C}_{\mathrm{m}}$ & 4.77 & 5.5 \\
& 6.96 & 22.1 \\
\hline \hline
\end{tabular}

and the two subsequent EPSR fits to these data using P1 and P2 reference potentials are shown in Fig. 3 and the coordination numbers are shown in Table VI. While both P1 and P2 reference potentials used for the EPSR models provide adequate fits to the data over most of the $Q$ range, there are marked deviations between the data and EPSR fit when using the P2 potential in the low- $Q$ region. Figure 4 shows the corresponding RDF's for DMSO from both EPSR models where the two potential models give somewhat different functions. The largest differences between the models are found in the $g_{\mathrm{SS}}(r), g_{\mathrm{SO}}(r)$, and $g_{\mathrm{OO}}(r)$ functions, indicating differences in the dipole-dipole correlations, since the dipole moment axis is collinear with the $\mathrm{S}-\mathrm{O}$ vector. Although the contribution of the heavier-atom RDF's to the total DMSO diffraction pattern is small (Table I) it is clear that the diffraction data are apparently sensitive to these functions. This sensitivity can be understood because of the molecular nature of the fluid and the severely constrained intramolecular configuration space; light-atom and heavy-atom correlations are therefore not independent of each other and are, in fact, strongly correlated. Because of this close relationship be-

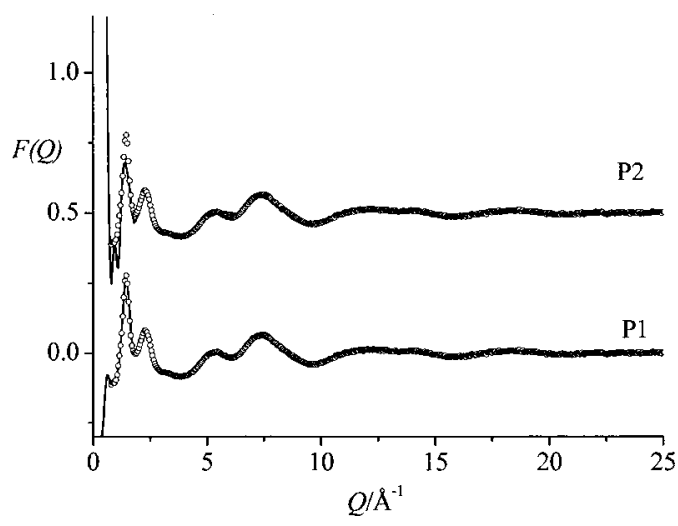

FIG. 3. $F(Q)$ data (circles) for $d_{6}$-DMSO at $T=298 \mathrm{~K}$ and subsequent fits to the data using the P1 and P2 potentials (solid lines) (Refs. 10 and 11), as reference potentials in the EPSR simulation. The P2 potential fit and data have been displaced by 0.5 for clarity.
TABLE VI. Coordination number for site-site radial distribution functions from EPSR fits to DMSO using the P1 reference potential (Refs. 10 and 11).

\begin{tabular}{clr}
\hline \hline $\mathrm{RDF}$ & $r(\AA)$ & $\mathrm{CN}$ \\
\hline $\mathrm{H}-\mathrm{H}$ & 2.09 & 0.5 \\
& 3.45 & 4.7 \\
$\mathrm{H}-\mathrm{C}$ & 4.05 & 1.3 \\
$\mathrm{H}-\mathrm{S}$ & 6.67 & 9.8 \\
$\mathrm{H}-\mathrm{O}$ & 3.382 & 1.3 \\
& 4.99 & 3.7 \\
$\mathrm{C}-\mathrm{O}$ & 4.62 & 2.8 \\
$\mathrm{~S}-\mathrm{O}$ & 3.81 & 1.2 \\
& 5.74 & 5.5 \\
$\mathrm{O}-\mathrm{O}$ & 4.85 & 3.2 \\
& 6.75 & 10.0 \\
$\mathrm{C}-\mathrm{C}$ & 4.53 & 4.5 \\
& 6.97 & 22.7 \\
$\mathrm{C}-\mathrm{S}$ & 6.11 & 7.6 \\
$\mathrm{~S}-\mathrm{S}$ & 4.75 & 2.6 \\
& 7.01 & 11.5 \\
\hline \hline
\end{tabular}

tween light-atom correlations, which dominate the diffraction pattern, and the heavier-atom correlations, which are much weaker in the data, the sensitivity of the simulation to heavy-atom correlations is therefore much greater than would be suggested by a simple, "atomic" picture of the fluid, where the Faber-Ziman coefficients alone would dictate the sensitivity.

Comparing the RDF's of the two measured fluids, the site-site radial distribution functions for both acetone models show similar trends to those seen in DMSO, but tend to be less structured in general. This is most notable in the $g_{\mathrm{C}_{c} \mathrm{O}}(r)$, $g_{\mathrm{C}_{c} \mathrm{C}_{c}}(r)$, and $g_{\mathrm{OO}}(r)$ functions (Fig. 2) which have similar shapes to the analogous $g_{\mathrm{SS}}(r), g_{\mathrm{SO}}(r)$, and $g_{\mathrm{OO}}(r)$ functions extracted from the P1 reference potential EPSR fits for DMSO. Though all of these RDF's are similar in shape to each other, the DMSO RDF's have more clearly defined peak

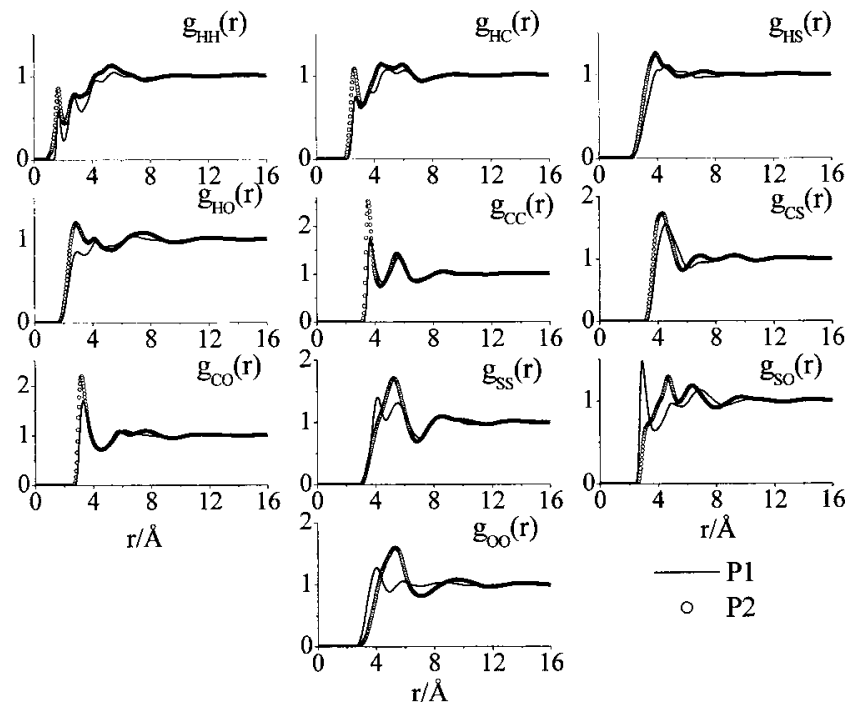

FIG. 4. Site-site intermolecular pair-correlation functions for DMSO generated by the EPSR fit to the data using the P1 potential (solid) and P2 potential (circles) (Refs. 10 and 11). 


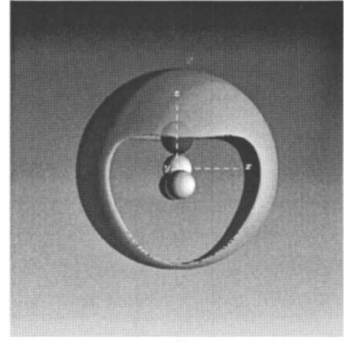

a)

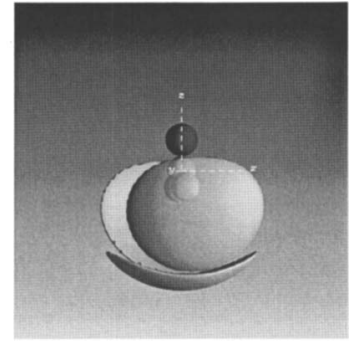

b)

FIG. 5. Spatial density functions for acetone generated by the EPSR fit to the data using the FHMK reference potential. (a) shows the neighboring molecules in the distance range of $2-5 \AA$ where the contour level has been chosen so that the surface shown encloses $70 \%$ of the neighboring molecules and (b) shows the neighboring molecules at the distance range of 5-7 $\AA$ where the contour level has been chosen so that the surface chosen encloses $15 \%$ of the molecules. In both pictures the size of the plotting box is $16 \AA$.

positions than the acetone fits; the peaks are sharper in these heavy-atom RDF's for DMSO, compared with the analogous functions for acetone.

As both the FHMK and WR EPSR models of acetone show virtually identical RDF's, the remainder of this paper shows only functions (SDF's and OCF's) extracted with the FHMK reference potential. Figure 5 shows two SDF's for acetone from this EPSR fit to the data. In both Figs. 5(a) and $5(\mathrm{~b})$, the central carbon $\left(\mathrm{C}_{\mathrm{C}}\right)$ of the acetone molecule, molecule 1 (described in Sec. III A 2), is located at the origin of the central axes. In keeping with the measured data, the acetone molecule retains its planar $C_{2 \nu}$ symmetry as the carbonyl bond is located along the $z$ axis while the methyl-methyl vector lies in the $y z$ plane (the methyl hydrogens have been omitted for clarity). Figure 5(a) shows the SDF where the surface contour encloses $70 \%$ of the molecules in the distance range of $2-5 \AA$. At this distance the majority of the nearest-neighbor molecules in liquid acetone are located either in a ring above the oxygen atom or along the plane of the molecule on both sides. Figure 5(b) shows the SDF for acetone in the second coordination shell at a distance of 5-7 $\AA$ where the surface contour encloses $15 \%$ of the molecules. Here the second shell shows the inverse of the first coordination shell with the highest density occurring perpendicular to the $x z$ plane of the central molecule as well as below the $x y$ plane.

Figure 6 shows the SDF's for DMSO from the EPSR fit using the P1 reference potential, where the sulfur atom on the central DMSO molecule is placed at the origin of the reference coordinate axes. As is the case with the carbonyl bond in acetone (Fig. 5), the sulfonyl bond is located along the $z$ axis; however, the methyl carbons are canted away from the $y z$ plane and lie below the $x y$ plane in order to give the correct pyramidal symmetry of the molecule, $C_{s}$. DMSO, in addition to retaining a different molecular geometry to acetone, shows a very different configuration both in the first and second coordination shells. In the first coordination shell, Fig. 6(a), from 2 to $5 \AA$ where again the surface contour encloses $70 \%$ of the molecules in this shell, the nearestneighbor molecules are found in a concave semicircular ring behind the central molecule at the apex of the intramolecular

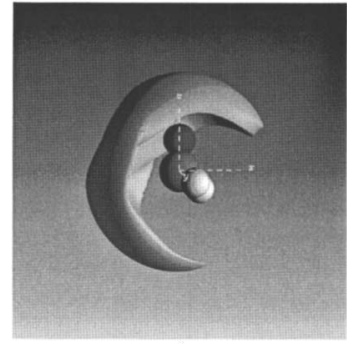

a)

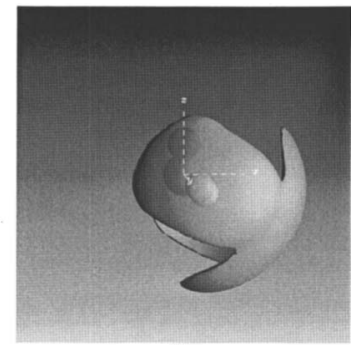

b)
FIG. 6. Spatial density functions for DMSO generated by the EPSR fit to the data using the P1 reference potential. (a) shows the neighboring molecules in the distance range of $2-5 \AA$ where the contour level has been chosen so that the plotted surface encloses $70 \%$ of the neighboring molecules and (b) shows the neighboring molecules at the distance range of 5-7 $\AA$ where the contour level has been chosen so that the plotted surface encloses $15 \%$ of the molecules. In both pictures the size of the plotting box is $16 \AA$.

"pyramid" above the central S atom. Also there is no density present below the $\left(\mathrm{CH}_{3}\right)_{2} \mathrm{SO}$ pyramid, signifying a low probability of locating nearest-neighbor molecules in this region of the first shell. As was the case with the acetone SDF's, the coordination in the second nearest-neighbor shell from 5 to $7 \AA$ with the surface contour enclosing $15 \%$ of the molecules in this shell in Fig. 6(b) shows the inverse of the first shell with the density being located in front of the central DMSO molecule as well as perpendicular to the $z x$ plane. Only the SDF's and OCF's (discussion below) generated using the P1 reference potential are shown as this model (Fig. 2) provides the best fit to the measured diffraction data.

A selection of the OCF's generated from the FHMK EPSR fit to the acetone data is shown in Fig. 7. The OCF's were extracted, as described in Sec. III A 2, as a function of
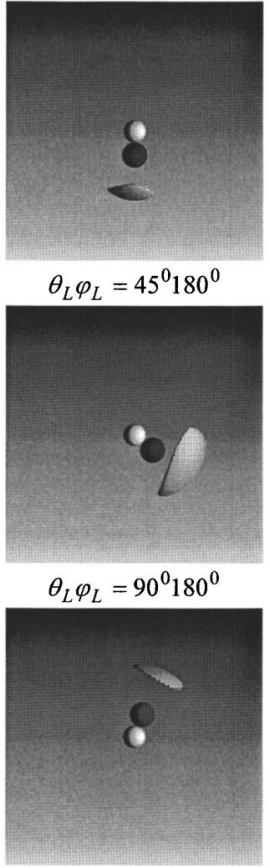

$\theta_{L} \varphi_{L}=135^{0} 180^{\circ}$

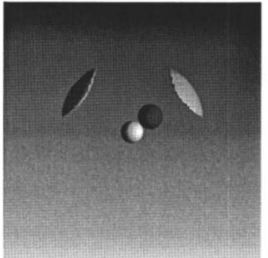

$\theta_{L} \varphi_{L}=0^{0} 0^{0}$
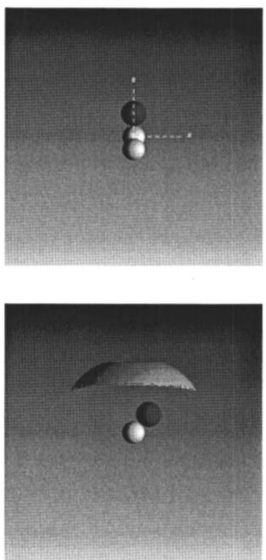

$\theta_{L} \varphi_{L}=180^{0} 0^{0}$

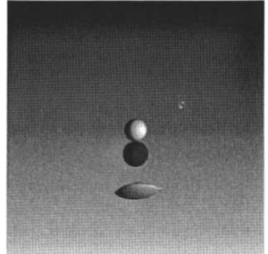

$\theta_{L} \varphi_{L}=45^{0} 0^{0}$

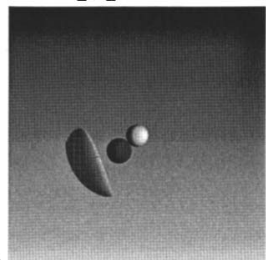

$\theta_{L} \varphi_{L}=90^{0} 0^{0}$

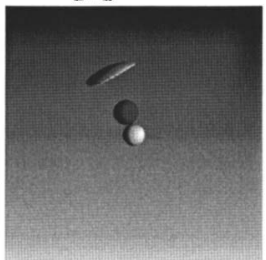

$\theta_{L} \varphi_{L}=135^{0} 0^{0}$
FIG. 7. Orientational correlations for acetone from the EPSR fits to the data using the FMHK potentials (Ref. 28). The contour level has been chosen so that the surface shown encloses $15 \%$ of the neighboring molecules in the distance range of $2-5 \AA$. In each case the size of the plotting box is $16 \AA$. 
three variables, $r, \varphi_{M}$, and $\theta_{M}$, by averaging over the $\chi_{M}$ angular coordinates where $\chi_{M}$ corresponds to the rotation of the methyl positions relative to the $\mathrm{C}=\mathrm{O}$ axis. In this figure the central panel shows an acetone molecule superimposed on the laboratory axes again without the hydrogen atoms. The surrounding panels show the OCF's plotted for a particular position relative to the central acetone molecule. As was described in detail in Sec. III A 2, the OCF can be extracted from the nearest-neighbor shell by probing the relative position of the second molecule (those located in the nearest-neighbor shell) to molecule 1 set at the origin of the laboratory axes where $\mathbf{r} \equiv\left(r, \omega_{L}\right) \equiv\left(r, \theta_{L} \varphi_{L}\right)$ defines the position of the molecules in the nearest-neighbor shell. Each panel shows the OCF at a particular location in the first coordination shell where each position shows a different $\omega_{L}$ value relative to the central axis and in each case $r=2-5 \AA$. For example, the panel in the top right-hand corner of Fig. 7 shows the most probable orientation of the next-nearestneighbor shell in liquid acetone at a position of $\omega_{L} \equiv \theta_{L} \varphi_{L}$ $=45^{\circ} 0^{\circ}$ where at this position the nearest-neighbor $\mathrm{C}=\mathrm{O}$ orientation is antiparallel to the central molecule. Because each of these orientational correlation functions has been averaged over rotations of the methyl positions about the central $\mathrm{C}=\mathrm{O}$ bond $\left(\chi_{M}\right)$ only the orientation of the $\mathrm{C}=\mathrm{O}$ bond relative to the central molecule is shown in each surrounding panel with the methyl groups omitted. Similar to the SDF's each OCF has a shell showing the most likely location of the nearest neighbor here relative to the central molecule at the coordinates indicated in the panel, $\omega_{L} \equiv \theta_{L} \varphi_{L}$. Where the SDF's show the density of the most probable location of molecules in the nearest-neighbor shell, the OCF shows the most probable orientation of nearest-neighbor molecules at a particular location in this shell. To show this pictorially, each panel in Fig. 7 surrounding the central molecule has the oxygen in the $\mathrm{C}=\mathrm{O}$ bond pointed toward the portion of the shell which shows the most probable orientation.

In the same manner as acetone, a selection of the OCF's - extracted by averaging over $\chi_{M}$ to give a threevariable $\left(r, \varphi_{M}\right.$, and $\left.\theta_{M}\right) \mathrm{OCF}$ - from the EPSR fit to DMSO data using the P1 reference potential is shown in Fig. 8. Again the surrounding panels show the OCF's plotted for a particular position relative to the central DMSO molecule $\left(\omega_{L} \equiv \theta_{L} \varphi_{L}\right)$ where in each case $r=2-5 \AA$. The most probable orientation in the nearest-neighbor shell is again shown by the $\mathrm{S}-\mathrm{O}$ bond of the surrounding molecules pointing toward the portion of the probability shell which shows the highest density. Only the $\mathrm{S}-\mathrm{O}$ bond is shown in each panel as each OCF function has been averaged over all methyl rotations, as described above.

\section{DISCUSSION}

\section{A. RDF's}

Given that the diffraction patterns in all cases are dominated by scattering from the hydrogen-containing partial structure factors (Table I), the hydrogen-containing RDF's for each liquid provide a reasonable picture of nearestneighbor contacts with respect to the methyl groups. The RDF's for intermolecular hydrogen contacts in acetone
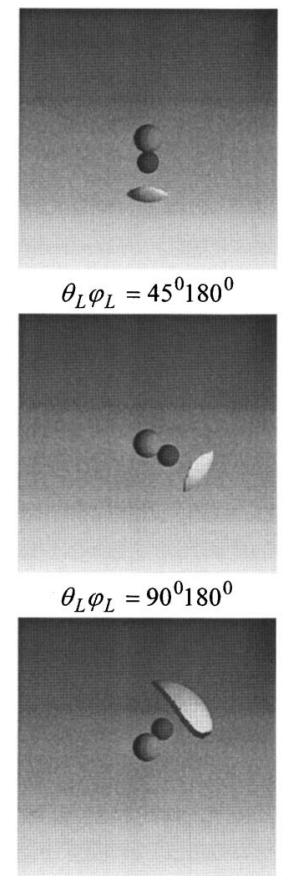

$\theta_{L} \varphi_{L}=135^{0} 180^{\circ}$

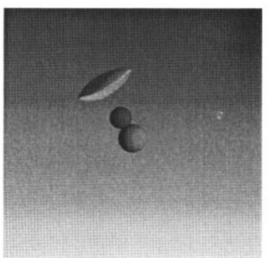

$\theta_{L} \varphi_{L}=0^{0} 0^{0}$
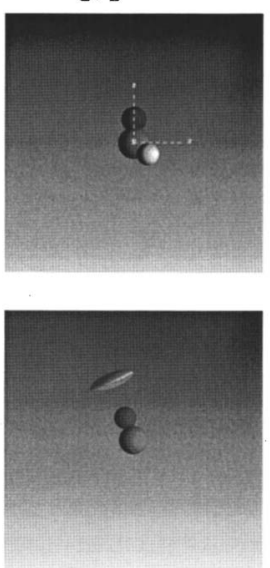

$\theta_{L} \varphi_{L}=180^{0} 0^{0}$

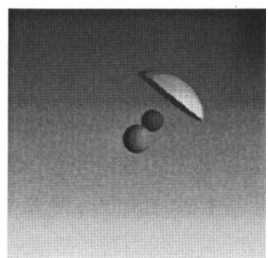

$\theta_{L} \varphi_{L}=45^{0} 0^{0}$

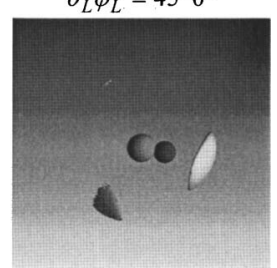

$\theta_{L} \varphi_{L}=90^{\circ} 0^{0}$

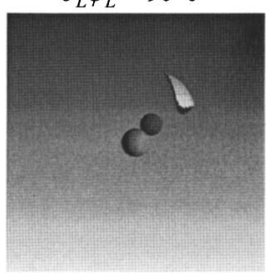

$\theta_{L} \varphi_{L}=135^{0} 0^{0}$

FIG. 8. Orientational correlations from the EPSR fits to the data using the P1 reference potential for DMSO (Ref. 10). The contour level has been chosen so that the surface shown encloses $15 \%$ of the neighboring molecules in the distance range of $2-5 \AA$. In each case the size of the plotting box is $16 \AA$.

(Fig. 2) each show a broad distribution of distances with the exception of the $g_{\mathrm{HC}_{c}}(r)$ function which shows a prominent peak at around $4 \AA$. Though this peak is clear evidence of an intermolecular $\mathrm{H} \cdots \mathrm{C}_{\mathrm{C}}$ contact, the distance is not sufficiently short to signify a hydrogen bond. Moreover, the presence of a $\mathrm{H} \cdots \mathrm{O}$ intermolecular contact is not discernable in the $g_{\mathrm{OH}}(r)$ function, indicating that there is no methyl hydrogen oxygen, $\mathrm{C}_{\mathrm{m}}-\mathrm{H} \cdots \mathrm{O}$, hydrogen bonding present in the liquid. It has been suggested that weak $\mathrm{C}_{\mathrm{m}}-\mathrm{H} \cdots \mathrm{O}$ contacts may "stabilize" the liquid structure, ${ }^{7}$ and these contacts have been linked to the anomalous heat-capacity behavior seen in solid-state acetone. ${ }^{9}$ Our simulations do not support this suggestion for the structure of acetone in the liquid state.

DMSO on the other hand shows more "structured" hydrogen RDF's irrespective of the reference potential used (Fig. 4). While this does not indicate hydrogen bonding per $s e$ it is indicative of preferred $\mathrm{H} \cdots X(X=\mathrm{C}$ or $\mathrm{O})$ intermolecular contacts in liquid DMSO, reminiscent of crystalline DMSO which contains intermolecular $\mathrm{O} \cdots \mathrm{H}$ distances of $2.40,2.51$, and $2.70 \AA{ }^{64}$ This is also supported by a recent $\mathrm{X}$-ray diffraction and molecular dynamics (MD) study of liquid DMSO which showed that the three nearest-neighboratoms to the oxygen atom were hydrogen. ${ }^{12}$ In a previous neutron diffraction study, these contacts were attributed to intramolecular distances; however, in that study it was not possible to distinguish between intra- and intermolecular contributions to the diffraction pattern. ${ }^{10}$ These contacts have previously been attributed to weak hydrogen bonds to the oxygen atoms in DMSO; ${ }^{34,35}$ though given the molecular nature of the system these contacts most likely arise from the dipole-dipole alignment of DMSO molecules in the fluid. 
The differences between the two models of DMSO, P1 and $\mathrm{P} 2$, are expected in the heavy atom RDF's given that the P1 potential does not have charge associated with the methyl groups while the $\mathrm{P} 2$ potential does include methyl charges (Table IV). Whereas the RDF's for acetone are virtually identical for each reference potential, the non-hydrogencontaining RDF's in DMSO provide a distinguishing difference between the two starting reference potentials. Specifically, in the $\mathrm{P} 2$ potential the methyl carbon has a charge of $q_{e}=0.160$ while the P1 potential eliminates this charge and assumes the dipole moment in DMSO to be associated with only the sulfur and oxygen atoms. The EPSR fit to the data using the P1 potential provides a much better fit and as such the RDF's generated with this potential provide a better representation of the local order in the data measured.

In the previous analysis of DMSO, ${ }^{10}$ the agreement between neutron diffraction data and MD simulations was determined by visual comparison to an extracted composite $S_{x x}(Q)$ function $(X=\mathrm{S}, \mathrm{O}$, and $\mathrm{C})$ from the neutron diffraction data. Both P1 and P2 showed a good agreement with the measured $\mathrm{HH}$ and $\mathrm{XH}$ functions ${ }^{10,11}$ while $\mathrm{P} 2$ showed a slightly worse agreement with the neutron-derived $X X$ function compared to $\mathrm{P} 1$, but was more accurate with regard to the thermodynamics of liquid DMSO. In the present study the diffraction data (Fig. 3) are, in fact, fit better by the EPSR method using the P1 reference potential, again showing as before that this model reproduces the structure more accurately than with the $\mathrm{P} 1$ potential. ${ }^{10}$

\section{B. Spatial density functions}

The SDF's for acetone (Fig. 5) and DMSO (Fig. 6) show different distributions in the first coordination sphere. In acetone there is an equal probability of the next nearest neighbor being located on either each side of the $x y$ plane or above the central molecule. DMSO, on the other hand, shows nearest-neighbor density located in a concave semicircle around the apex of the pyramid where the $\mathrm{S}$ atom is located. The differences in the SDF's of these two liquids can be attributed largely to the difference in molecular geometries of the two molecules. The pyramidal molecular geometry of DMSO prevents molecules from approaching each other underneath the pyramid very easily while in acetone, which is planar, there is an equal probability of finding other molecules on each side of the central molecule in the $y z$ plane. Although the two molecules show vastly different densities in the $y z$ and $x y$ planes of the extracted SDF's, the liquids are similar in the respect that there is a high probability of nearest-neighbor molecules being located directly above the oxygen atom of the central molecule: in each liquid there is clear density in both Figs. 5 and 6 above the $z$ axis.

We also note that the arrangement of neighbors around the methyl groups in both molecules is qualitatively similar: the only real difference is that in acetone this arrangement lies symmetric about the $y z$ plane, while in DMSO it is rotated about the molecular $y$ axis to around $45^{\circ}$ along the positive $x$ axis, corresponding to the rotated positions of the methyl groups in DMSO compared to acetone. In both cases the methyl group coordination consists of a central band of neighbors which passes between the two methyl head groups, and a shell of neighbors around each individual methyl head group.

\section{Orientational correlation functions}

A direct picture of the dipole-dipole interactions present in the first coordination sphere of liquid acetone and DMSO can be seen by the orientational plots shown in Figs. 7 and 8, respectively.

\section{Acetone OCF's}

In acetone, Fig. 7, the dipole orientation in the nearestneighbor shell directly in front and behind the $z y$ plane of the central molecule at $\theta_{L} \varphi_{L}=45^{\circ} 0^{\circ}$ and $\theta_{L} \varphi_{L}=45^{\circ} 180^{\circ}$ shows an antiparallel alignment (with the oxygen atom pointing downwards) relative to the central molecule. This dipole interaction "flips" to a parallel alignment at $\theta_{L} \varphi_{L}=135^{\circ} 0^{\circ}$ and at $\theta_{L} \varphi_{L}=135^{\circ} 180^{\circ}$ again on both sides of the central molecule, with the oxygen atom pointing upwards towards the central acetone molecule at these locations in the first coordination sphere. Between these two locations at $\theta_{L} \varphi_{L}$ $=90^{\circ} 0^{\circ}$ and $\theta_{L} \varphi_{L}=90^{\circ} 180^{\circ}$ there is a transition between antiparallel and parallel alignments. At each position in the first coordination shell in front or behind the plane of the central molecule, the oxygen atom is pointed toward this central molecule.

The preferred orientation in the first coordination sphere directly above $\left(\theta_{L} \varphi_{L}=0^{\circ} 0^{\circ}\right)$ the central acetone molecule is with the $\mathrm{C}=\mathrm{O}$ bond pointed away from the central molecule with the second nearest neighbor canted either to the right or left while below the central molecule $\left(\theta_{L} \varphi_{L}=180^{\circ} 0^{\circ}\right)$ the orientation of the dipole is parallel with a "ring" of different distributions all with the $\mathrm{C}=\mathrm{O}$ pointed toward the central molecule. Upon inspection of the first coordination shell SDF for acetone (Fig. 5) it is evident that there is a low probability of finding nearest-neighbor molecules directly below the central molecule.

Figure 7 shows evidence of preferred orientation in the first coordination shell of liquid acetone. The symmetric orientations seen on both sides of the central acetone molecule are expected, given that acetone is symmetric with respect to both the $x z$ and $y z$ planes. Additionally, this behavior in the first coordination shell is observed in a comparative simulation and integral equation study by Richardi et al. ${ }^{31}$ It is also of note that early diffraction measurements of the liquid, where orientational correlations were not directly determined, ${ }^{7}$ predicted predominantly perpendicular dipole interactions in the first coordination shell of acetone. Here, given that the most likely location of molecules in the nearest-neighbor shell is around the oxygen atom of the central molecule, the average would more likely show an antiparallel configuration. This is similar to the crystalline structure where the nearest-neighbor molecules are aligned as dimers in an antiparallel configuration. ${ }^{9}$ The top panel in Fig. 7 shows the most probable location of dipole-dipole alignment with the $\mathrm{C}=\mathrm{O}$ bond diagonal to the central molecule and the oxygen atom pointing away from the center around the ring shown in this figure. Though the exact methyl ori- 

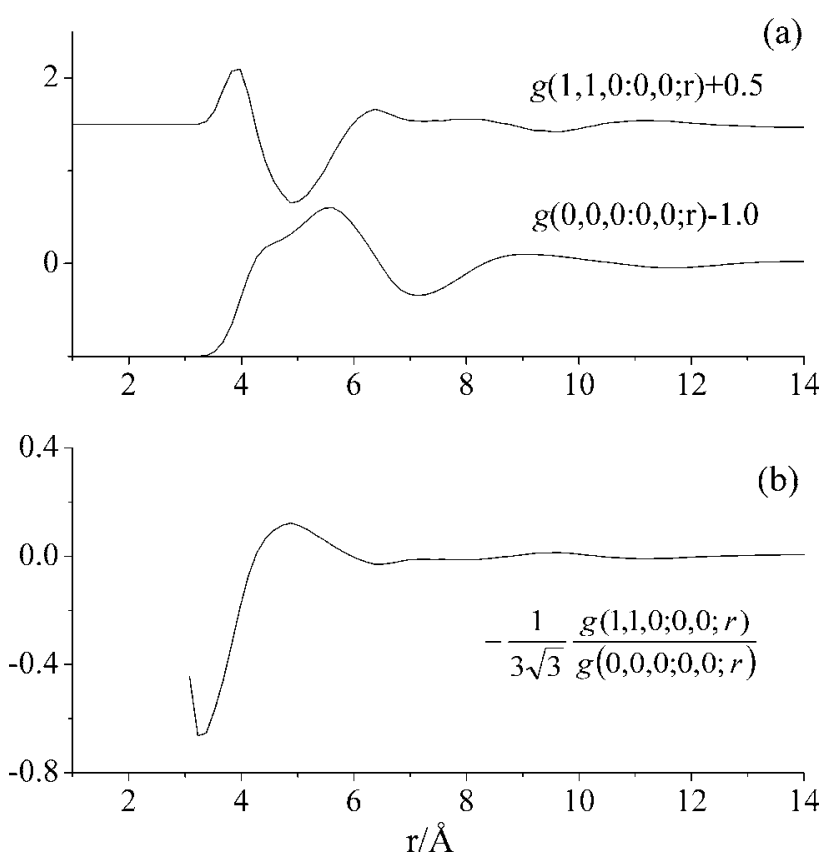

FIG. 9. (a) $g(000 ; 00 ; r)+0.5$ and $g(110 ; 00 ; r)-1$ functions for acetone. For clarity, the coefficients are shown as $g(110 ; 00 ; r)-1$ and $g(110 ; 00 ; r)$ +1.0. (b) The ratio $-(1 / 3 \sqrt{3})[g(110 ; 00 ; r) / g(000 ; 00 ; r)]$, which represents the average cosine between dipoles in acetone as a function of molecular separation.

entations are unspecified in the present plots (because the correlation functions shown in this figure have been averaged over rotations about the $\mathrm{C}=\mathrm{O}$ bond for ease of presentation), this implies that there is an interaction between the methyl groups and the oxygen atom at this location. This interaction is probably weak and does not show formal hydrogen bonding, given the absence of any obvious structure in the hydrogen-containing RDF's for acetone (Fig. 3); however, it does resemble the solid-state structure of acetone where $\mathrm{C}_{\mathrm{m}}-\mathrm{H} \cdots \mathrm{O}$ contacts are thought to stabilize the structure. ${ }^{9}$

Given that the graphs of Fig. 7 show only the orientations of the top $15 \%$ molecules in the distance range specified, it might be concluded that the orientational correlations in acetone are weak. In order to assess the strength of the orientation correlations in acetone, Fig. 9(a) shows the $g(000 ; 00 ; r)$ and $g(110 ; 00 ; r)$ coefficients obtained from the spherical harmonic expansion of the orientational paircorrelation function (Sec. III A 2). The former function represents the center-center radial distribution function between molecules, and the latter, when multiplied by $C(110: 00) / 3$ $=-1 / 3 \sqrt{3}$, represents the distribution of the cosine of the angle between dipole moment vectors on pairs of molecules, $\left\langle\boldsymbol{\Omega}_{1} \cdot \boldsymbol{\Omega}_{2} /\left|\Omega_{1}\right|\left|\Omega_{2}\right|\right\rangle$, as a function of separation. ${ }^{62}$ The ratio $-(1 / 3 \sqrt{3})[g(110 ; 00 ; r) / g(000 ; 00 ; r)]$, Fig. 9(b), therefore represents the mean value of this cosine for a pair molecules separated by a distance $r$. The values of this function in the region of the first neighbor peak are not those of weakly correlated orientations. Indeed the orientations are strongly correlated in the first neighbor shell, and flip from being strongly antiparallel at short distances to more parallel slightly further out.

\section{DMSO OCF'S}

The pyramidal geometry ensures that, distinct from acetone, DMSO is only symmetric with respect to the $x z$ plane and as such the dipole-dipole orientations in the first coordination shell (Fig. 8) vary at different positions in front and behind the plane of the central molecule. Behind the central molecule in the $z y$ plane, DMSO molecules in the first coordination shell show a head-to-tail dipole $\mathrm{S}-\mathrm{O} \cdots \mathrm{S}-\mathrm{O}$ alignment at all positions $\left(\theta_{L} \varphi_{L}=45^{\circ} 180^{\circ}, \theta_{L} \varphi_{L}=90^{\circ} 180^{\circ}\right.$, and $\left.\theta_{L} \varphi_{L}=135^{\circ} 180^{\circ}\right)$ where in each case the oxygen atom in these nearest-neighbor molecules points toward the sulfur atom in the central DMSO molecule, analogous to what happens in acetone. Directly in front of the central DMSO pyramid, in the $z y$ plane $\left(\theta_{L} \varphi_{L}=45^{\circ} 0^{\circ}, \theta_{L} \varphi_{L}=90^{\circ} 0^{\circ}\right.$, and $\theta_{L} \varphi_{L}$ $\left.=135^{\circ} 0^{\circ}\right)$ the orientation of molecules in the surrounding shell all shows the $\mathrm{S}-\mathrm{O}$ bond pointing away from the $\mathrm{S}-\mathrm{O}$ bond in the central molecule. It should be noted that at $\theta_{L} \varphi_{L}=90^{\circ} 0^{\circ}$ and $\theta_{L} \varphi_{L}=135^{\circ} 0^{\circ}$ the likelihood of nearestneighbor molecules being present here is quite low given the absence of density seen in the SDF (Fig. 6) at these locations.

Above the central DMSO molecule $\left(\theta_{L} \varphi_{L}=0^{\circ} 0^{\circ}\right)$ the orientation of nearest-neighbor molecules shows a preference for the $\mathrm{S}-\mathrm{O}$ bond to point away from the oxygen atom in the central molecule while below the molecule $\left(\theta_{L} \varphi_{L}=180^{\circ} 0^{\circ}\right)$ the $\mathrm{S}-\mathrm{O}$ bonds of the nearest-neighbor molecule point toward the central sulfur atom at the origin of the central molecule.

In the first coordination sphere, it is evident at each location that the most probable alignment of the $\mathrm{S}-\mathrm{O}$ bonds of the surrounding molecules is in a head-to-tail fashion with the $\mathrm{S}-\mathrm{O}$ bond of the central molecule. From Fig. 9, it is clear that there is a $\mathrm{S}-\mathrm{O} / \mathrm{S}-\mathrm{O}$ alignment between the central molecule and the surrounding molecules in the shell.

It has been asserted in the literature that the crystalline structure is partially retained in liquid DMSO, which shows antiparallel interactions between DMSO molecules. ${ }^{6,12,64}$ Here the only antiparallel configuration is behind the $\mathrm{S}-\mathrm{O}$ bond of the central molecule at $\theta_{L} \varphi_{L}=45^{\circ} 180^{\circ}$. The antiparallel configuration of DMSO molecules from the crystal structure is not retained in the liquid. As stated above at all positions in the first coordination shell the molecular alignment shows "head-to-tail" $\mathrm{S}-\mathrm{O} / \mathrm{S}-\mathrm{O}$ interactions. This type of dipole-dipole preference in liquid DMSO is predicted by simulation $^{34,35}$ and is found to have a local minima in density functional theory (DFT) calculations on DMSO-DMSO dimers. ${ }^{12,38}$

Though the dipole-dipole orientations in liquid DMSO and acetone are different in many respects they also show certain similarities, the dipole orientations directly above both molecules both show the $X-\mathrm{O}\left(X=\mathrm{S}\right.$ or $\left.\mathrm{C}_{\mathrm{C}}\right)$ bond pointing away from the central molecule. In acetone there is a broad distribution of $\mathrm{C}=\mathrm{O}$ orientations above the molecule while in DMSO the $\mathrm{S}-\mathrm{O}$ bond is in a more specific location. In both cases this implies some $\mathrm{C}_{\mathrm{m}}-\mathrm{H} \cdots \mathrm{O}$ interactions where this interaction is more structured in DMSO, as evidenced by a comparison of the hydrogen-containing RDF's. Although neither of these liquids show an intermediate range 

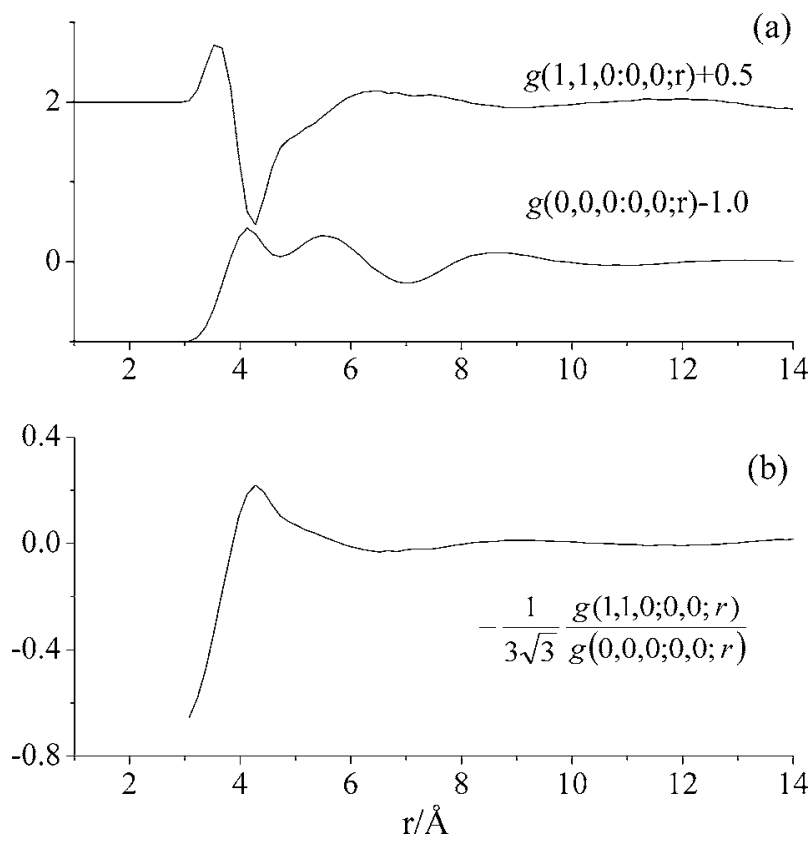

FIG. 10. (a) $g(000 ; 00 ; r)$ and $g(110 ; 00 ; r)$ functions for DMSO. For clarity, the coefficients are shown as $g(000 ; 00 ; r)-1$ and $g(110 ; 00 ; r)+1.0$. (b) The ratio $-(1 / 3 \sqrt{3})[g(110 ; 00 ; r) / g(000 ; 00 ; r)]$, which represents the average cosine between dipoles in DMSO as a function of molecular separation.

order, it is apparent that there is some ordering of dipoles in the first coordination shell as a proportion of the molecules show dipole-induced alignments.

As for acetone, the mean dipole-dipole cosine for DMSO is shown in Fig. 10. Here it is seen that the orientational correlations are even stronger in the first shell than for acetone. Again a strong antiparallel alignment is seen at distances shorter than the main near-neighbor peak. Beyond that the alignment becomes more parallel, as discussed with respect to Fig. 9, but the size of the mean cosine indicates that these orientational correlations are strong, even if complex in nature.

\section{CONCLUSIONS}

The problem of extracting reliable structural information about molecular arrangement and orientation in relatively simple molecular fluids such as the present examples is well known. ${ }^{63}$ Unlike the case of water of $\mathrm{HF}^{50,52}$ where the diffraction data can be separated into the complete set of partial structure factors, this is not possible to achieve in most cases likely to be of chemical or biological interest. The present experiment and analysis have relied heavily on EPSR to fill in the gaps of missing structural information by imposing reasonable constraints on atomic overlap and on the likely molecular geometries. By comparing the model and data directly in the reciprocal space of the measurements, one has the best chance of avoiding the spurious structures and conclusions that might be generated by systematic effects in the data (such as inelasticity effects with neutron scattering or Compton scattering with $\mathrm{x}$ rays). We believe the present comparison in $Q$ space using the total differential scattering cross sections as measured, rather than the derived functions, is likely to give a more accurate representation of the local order in molecular fluids. In particular, the orientational information contained in Figs. 7-10 will be proven highly informative in more complex systems for identifying the local molecular order.

Through a detailed study of the orientational correlations found from EPSR fits to measured neutron diffraction data it is found that DMSO and acetone show some similarities but also marked differences between their nearest-neighbor dipole alignment in the liquid. The structure of the first shell is strongly influenced by the shape of the molecule, with a predominance of close contacts around the oxygen atom of both molecules, and much weaker correlations at a larger distance around the methyl head groups. This distribution follows the underlying symmetry of the central molecule, with a symmetric distribution of neighbors found around the acetone molecule, and a strongly asymmetric distribution found around the pyramidal DMSO molecule, Figs. 5 and 6. There is also a high degree of preferred orientation shown in the first coordination shell in each liquid, Figs. 7 and 8. Both liquids show a predominance of antiparallel alignments of molecular dipole moments at short distances inside the main nearest-neighbor peak, while at larger distances they become more parallel, adopting a range of orientations. In acetone these arrangements are symmetric about the central plane of the acetone molecule, but in DMSO the antiparallel arrangement is found only behind the molecule, with a more parallel or tangential orientation in front of the molecule, a region which, however, is only sparsely populated in the first shell. A general rule for both molecules is that outside the antiparallel arrangement seen at short distances, the molecules adopt more of a head-to-tail configuration, reminiscent of what might occur due to dipole ordering over longer-distance scales. This ordering is, however, tempered by the underlying symmetry of the molecule. The alignment seen in acetone would not be possible in DMSO given its pyramidical molecular geometry. Additionally there is evidence for weak intermolecular $\mathrm{C}_{\mathrm{m}}-\mathrm{H} \cdots \mathrm{O}$ contacts in both fluids.

\section{ACKNOWLEDGMENTS}

The authors would like to thank U.S. National Science Foundation for financial support for one of the authors (S.E.M.) under Award No. OISE-0404938 and for another author (A.L.) under Award Nos. CHE-0211626 and CHE0512131.

${ }^{1}$ J. March and M. B. Smith, March's Advanced Organic Chemistry: Reactions, Mechanisms and Structure, 5th ed. (Wiley, New York, 2000).

${ }^{2}$ Handbook of Chemistry and Physics, 83rd ed. (CRC, New York, 2002).

${ }^{3}$ H. Dreizler and G. Dendl, Z. Naturforsch. A 19A, 512 (1964).

${ }^{4}$ F. A. Cotton and R. Francis, J. Am. Chem. Soc. 82, 1986 (1960).

${ }^{5}$ R. J. Gillespie and E. A. Robinson, Angew. Chem., Int. Ed. Engl. 35, 495 (1996).

${ }^{6}$ H. Bertagnolli and P. Chieux, Phys. Chem. 93, 88 (1989).

${ }^{7}$ H. Bertagnolli, M. Hoffman, and M. Ostheimer, Z. Phys. Chem. 165, 165 (1989).

${ }^{8}$ H. Bertagnolli, M. Hoffman, and P. Chieux, Z. Phys. Chem. 159, 185 (1988).

${ }^{9}$ D. R. Allan, S. J. Clark, R. M. Ibberson, S. Parsons, C. R. Pulham, and L. Sawyer, Chem. Commun. (Cambridge) 1999, 751.

${ }^{10}$ A. Luzar, A. K. Soper, and D. Chandler, J. Chem. Phys. 99, 6836 (1993).

${ }^{11}$ A. Luzar and D. Chandler, J. Chem. Phys. 98, 8160 (1993).

${ }^{12}$ U. Onthong, T. Megyes, I. Bako, T. Radnai, T. Grosz, K. Hermansson, 
and M. Probst, Phys. Chem. Chem. Phys. 6, 2136 (2004).

${ }^{13}$ J. D. Swalen and C. C. Costain, J. Chem. Phys. 31, 1562 (1959).

${ }^{14}$ R. Nelson and L. Pierce, J. Mol. Spectrosc. 18, 344 (1965).

${ }^{15}$ O. Bastiansen and H. Viervoll, Acta Chem. Scand. (1947-1973) 2, 702 (1948).

${ }^{16}$ D. B. Chesnut, J. Am. Chem. Soc. 120, 10504 (1998).

${ }^{17}$ J. Cioslowski and S. T. Mixon, Inorg. Chem. 32, 3209 (1993).

${ }^{18}$ T. P. Cunningham, D. L. Cooper, J. Gerratt, P. B. Karadakov, and M. Rairnondi, J. Chem. Soc., Faraday Trans. 93, 2247 (1997).

${ }^{19}$ A. E. Reed and V. R. Schleyer, J. Am. Chem. Soc. 112, 1434 (1990).

${ }^{20}$ K. K. Kelley, J. Am. Chem. Soc. 51, 1145 (1929).

${ }^{21}$ A. K. Soper and A. Luzar, J. Chem. Phys. 97, 1320 (1992).

${ }^{22}$ Y. Koga, Y. Kasahara, K. Yoshino, and K. Nishikawa, J. Solution Chem. 30, 885 (2001).

${ }^{23}$ S. Itoh and H. Ohtaki, Z. Naturforsch., A: Phys. Sci. 42, 858 (1987).

${ }^{24}$ A. K. Soper and A. Luzar, J. Phys. Chem. 100, 1357 (1996).

${ }^{25}$ E. Bernardi and H. Stassen, J. Chem. Phys. 120, 4860 (2004).

${ }^{26}$ I. A. Borin and M. S. Skaf, J. Chem. Phys. 110, 6412 (1999).

${ }^{27}$ M. Ferrario, M. Haughney, I. R. McDonald, and M. J. Klein, J. Chem. Phys. 93, 5156 (1990).

${ }^{28}$ L. C. G. Freitas, J. M. M. Cordeiro, and F. L. L. Garbajo, J. Mol. Liq. 79, 1 (1999).

${ }^{29}$ B. Kirchner and J. Hutter, Chem. Phys. Lett. 364, 497 (2002).

${ }^{30}$ R. L. Mancera, M. Chalaris, K. Refson, and J. Samios, Phys. Chem. Chem. Phys. 6, 94 (2004).

${ }^{31}$ J. Richardi, P. H. Fries, R. Fischer, S. Rast, and H. Krienke, Mol. Phys. 93, 925 (1998).

${ }^{32}$ M. S. Skaf, J. Phys. Chem. A 103, 10719 (1999).

${ }^{33}$ A. Vishnyakov, A. P. Lyubartsev, and A. Laaksonen, J. Phys. Chem. A 105, 1702 (2001)

${ }^{34}$ B. Rao and U. Singh, J. Am. Chem. Soc. 112, 3802 (1990).

${ }^{35}$ I. Vasiman and M. Berkowitz, J. Am. Chem. Soc. 114, 7889 (1992).

${ }^{36}$ H. Liu, F. Muller-Plathe, and W. van Gusteren, J. Am. Chem. Soc. 117, 4363 (1995).

${ }^{37}$ A. Ayda, O. Kalugin, M. Volobuev, and Y. Kolesnik, Mol. Phys. 99, 835 (2001).

${ }^{38}$ J. M. M. Cordeiro, Mol. Eng. 8, 303 (1999).

${ }^{39}$ M. L. Strader and S. E. Feller, J. Phys. Chem. A 106, 1074 (2002).

${ }^{40}$ D. R. Wheeler and R. L. Rowley, Mol. Phys. 94, 555 (1998).
${ }^{41}$ T. Radnai, I. Bako, P. Jedlovszky, and G. Palinkas, Mol. Simul. 16, 345 (1996).

${ }^{42}$ P. Jedlovszky and G. Palinkas, Mol. Phys. 84, 217 (1995).

${ }^{43}$ W. L. Jorgensen, J. M. Briggs, and M. L. Contreras, J. Phys. Chem. 94, $1683(1990)$

${ }^{44}$ S. Weerasinghe and P. E. Smith, J. Chem. Phys. 118, 10663 (2003).

${ }^{45}$ D. S. Venables and C. A. Schmuttenmaer, J. Chem. Phys. 113, 11222 (2000).

${ }^{46}$ Q. F. Ahkong, D. Fischer, W. Tampion, and J. A. Lucy, Nature (London) 253, 194 (1975).

${ }^{47}$ D. Martin and H. G. Hanthal, Dimethyl Sulfoxide (Wiley, New York, 1975).

${ }^{48}$ A. K. Soper, Mol. Phys. 99, 1503 (2001).

${ }^{49}$ J. L. Finney, A. K. Soper, and J. Turner, Physical Chemistry of Aqueous Systems, 12th Proceedings of the International Conference on the Properties of Water Steam, 1995, p. 468.

${ }^{50}$ A. K. Soper, Chem. Phys. 258, 121 (2000).

${ }^{51}$ A. K. Soper and P. A. Egelstaff, Mol. Phys. 42, 399 (1981).

${ }^{52}$ S. E. McLain, C. J. Benmore, J. E. Siewenie, J. Urquidi, and J. F. C. Turner, Angew. Chem., Int. Ed. 43, 1952 (2004).

${ }^{53}$ J. L. Finney, D. T. Bowron, and A. K. Soper, J. Phys.: Condens. Matter 12, A123 (2000).

${ }^{54}$ D. T. Bowron, J. L. Finney, and A. K. Soper, Mol. Phys. 93, 531 (1998).

${ }^{55}$ C. Andreani, M. Nardone, F. P. Ricci, and A. K. Soper, Phys. Rev. A 46, 4709 (1992)

${ }^{56}$ C. Andreani, F. Menzinger, M. A. Ricci, A. K. Soper, and J. Dreyer, Phys. Rev. B 49, 3811 (1994).

${ }^{57}$ C. Andreani, V. Merlo, M. A. Ricci, and A. K. Soper, Mol. Phys. 73, 407 (1991).

${ }^{58}$ V. F. Sears, Neutron News 3, 29 (1992).

${ }^{59}$ A. K. Soper, W. S. Howells, and A. C. Hannon, "ATLAS" Analysis of time-of-flight diffraction data from liquid and amorphous samples, R.A.L. Report No.RAL-89-046, 1989.

${ }^{60}$ I. M. Svishchev and P. G. Kusalik, J. Chem. Phys. 99, 3049 (1993).

${ }^{61}$ I. M. Svishchev and P. G. Kusalik, Chem. Phys. Lett. 215, 596 (1993).

${ }^{62}$ A. K. Soper, J. Chem. Phys. 101, 6888 (1994).

${ }^{63}$ C. G. Gray and K. E. Gubbins, Theory of Molecular Liquids (Oxford University Press, New York, 1984), Vol. I.

${ }^{64}$ R. Thomas, C. B. Shoemaker, and K. Eriks, Acta Crystallogr. 21, 12 (1966). 